YITP-SB-03-63

gr-qc/0312021

\title{
The Petrov type of the five-dimensional Myers-Perry metric
}

\author{
Pieter-Jan De Smet \\ C. N. Yang Institute of Theoretical Physics \\ State University of New York \\ Stony Brook, NY 11794-3840, USA \\ E-mail: Pieterj@insti.physics.sunysb.edu
}

\begin{abstract}
We point out that the Myers-Perry metric in five dimensions is algebraically special. It has Petrov type $\underline{22}$, which is the Petrov type of the five-dimensional Schwarzschild metric.

Keywords: Classical Theories of Gravity, Black Holes.
\end{abstract}




\section{Contents}

$\begin{array}{ll}\text { 1. Introduction } & 1\end{array}$

2. Review of the five-dimensional Petrov classification $\quad 1$

3. The Myers-Perry metric has Petrov type $\underline{22} \quad 2$

4. Conclusions and topics for further research $\quad 3$

\section{Introduction}

In this article, we calculate the Petrov type of the five-dimensional Myers-Perry metric [1]. The five-dimensional Myers-Perry (MP) metric is the generalization to five dimensions of the four-dimensional Kerr-metric. The Kerr-metric, which describes the gravitational field of a rotating star, and its static limit, the Schwarzschild metric, have both Petrov type $D$. It is remarkable to see that, although the Kerr-metric has fewer symmetries than the Schwarzschild metric, it does still have the same Petrov type.

We show that the same holds in five dimensions. The five-dimensional MP metric, which describes a rotating black hole in five dimensions, and its static limit, the five-dimensional Schwarzschild metric, have both Petrov type $\underline{22}$. Again, we see that although the fivedimensional MP metric has fewer isometries than the five-dimensional Schwarzschild metric, it does have the same Petrov type. The remainder of the article is organized as follows. In Section 2, we give a review of the five-dimensional Petrov classification. The Petrov type of the MP metric is given in Section 3. We conclude in Section 4.

\section{Review of the five-dimensional Petrov classification}

We only give a brief review of this classification, a longer discussion can be found in ref. [2]. We need to introduce two objects, the Weyl spinor and the Weyl polynomial. The Weyl spinor $\Psi_{a b c d}$ is the spinorial translation of the Weyl tensor $C_{i j k l}$

$$
\Psi_{a b c d}=\left(\gamma_{i j}\right)_{a b}\left(\gamma_{k l}\right)_{c d} C^{i j k l}
$$

Here, $\gamma_{i j}=\frac{1}{2}\left[\gamma_{i}, \gamma_{j}\right]$, where $\gamma_{i}$ are the $\gamma$-matrices in five dimensions. In this article, we use the following representation $\gamma_{1}=i \sigma_{1} \otimes 1, \gamma_{2}=\sigma_{2} \otimes 1, \gamma_{3}=\sigma_{3} \otimes \sigma_{1}, \gamma_{4}=\sigma_{3} \otimes \sigma_{2}$ and 


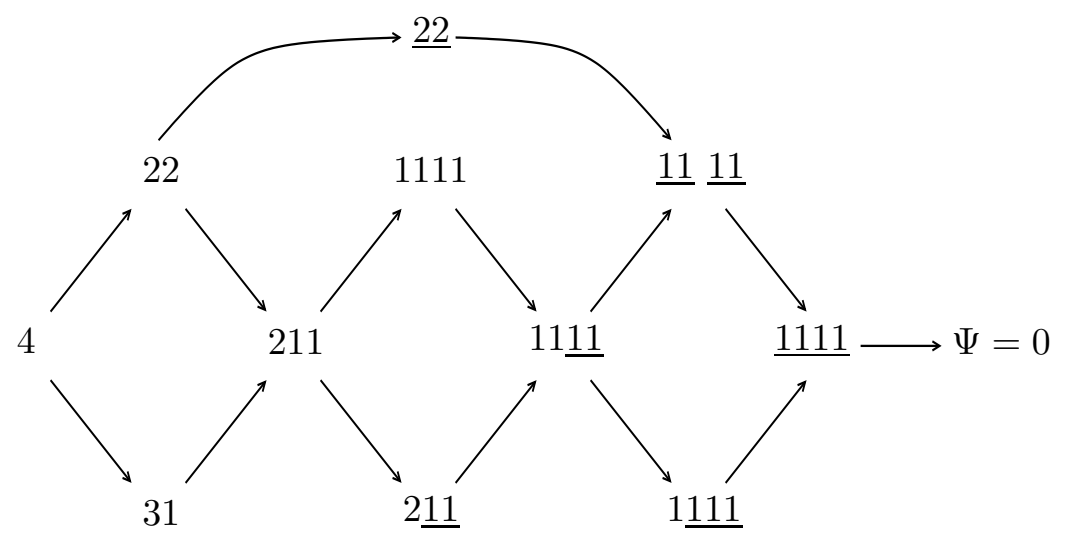

Figure 1: The 12 different Petrov types in 5 dimensions.

$\gamma_{5}=\sigma_{3} \otimes \sigma_{3}$. The Weyl spinor is symmetric in all its indices. The Weyl polynomial $\Psi$ is a homogeneous polynomial of degree four in four variables:

$$
\Psi=\Psi_{a b c d} x^{a} x^{b} x^{c} x^{d}
$$

The Petrov type of a given Weyl tensor is the number and multiplicity of the irreducible factors of its corresponding Weyl polynomial $\Psi$. In this way, we obtain 12 different Petrov types, which are depicted in figure 1 . We use the following notation. The number denotes the degree of the irreducible factors and underbars denote the multiplicities. For example, a Weyl polynomial which can be factorized into two different factors, each having degree 2, has Petrov type 22. If the two factors of degree 2 are the same, the Petrov type is $\underline{22}$.

\section{The Myers-Perry metric has Petrov type $\underline{22}$}

The five-dimensional rotating black hole is described by the Myers-Perry metric [1]

$$
\begin{aligned}
d s^{2}= & -d t^{2}+\frac{2 m}{\rho^{2}}\left[d t-a \sin ^{2} \theta d \phi-b \cos ^{2} \theta d \psi\right]^{2} \\
& +\frac{\rho^{2}}{R^{2}} d r^{2}+\rho^{2} d \theta^{2}+\Sigma_{a}^{2} \sin ^{2} \theta d \phi^{2}+\Sigma_{b}^{2} \cos ^{2} \theta d \psi^{2}
\end{aligned}
$$

where $\rho^{2}=r^{2}+a^{2} \cos ^{2} \theta+b^{2} \sin ^{2} \theta, \Sigma_{a}^{2}=r^{2}+a^{2}, \Sigma_{b}^{2}=r^{2}+b^{2}$ and

$$
R^{2}=\left[\Sigma_{a}^{2} \Sigma_{b}^{2}-2 m r^{2}\right] / r^{2}
$$


We choose the following tetrad

$$
\begin{aligned}
e_{1} & =\frac{1}{r^{2} R \rho}\left[\Sigma_{a}^{2} \Sigma_{b}^{2} \partial_{t}+a \Sigma_{b}^{2} \partial_{\phi}+b \Sigma_{a}^{2} \partial_{\psi}\right] \\
e_{2} & =\frac{1}{r \rho \sin \theta}\left[\Sigma_{b}\left(\partial_{\phi}+a \sin ^{2} \theta \partial_{t}\right)+b \Sigma_{a} V\right] \\
e_{3} & =\frac{1}{r \rho \cos \theta}\left[\Sigma_{a}\left(\partial_{\psi}+b \cos ^{2} \theta \partial_{t}\right)-a \Sigma_{b} V\right] \\
e_{4} & =\frac{R}{\rho} \partial_{r} \\
e_{5} & =\frac{1}{\rho} \partial_{\theta} .
\end{aligned}
$$

Here, we have used the vectorfield

$$
V=\frac{1}{\Sigma_{a} \Sigma_{b}+r \rho}\left(a \sin ^{2} \theta \partial_{\psi}-b \cos ^{2} \theta \partial_{\phi}\right)
$$

A straigthforward calculation gives the Weyl polynomial

$$
\Psi=-\frac{48 m r^{2}}{\rho^{6}}\left[x^{2}-y^{2}+z^{2}-t^{2}-2 f(x y+z t)-2 i g(x z+y t)\right]^{2},
$$

where

$$
\begin{aligned}
& f=\frac{b \sin \theta}{r} \frac{a^{2} \Sigma_{b} \rho+\left(b^{2}-a^{2}\right) r \Sigma_{a} \cos ^{2} \theta}{\Sigma_{a}^{2} \Sigma_{b}^{2}-r^{2} \rho^{2}}, \\
& g=\frac{a \cos \theta}{r} \frac{b^{2} \Sigma_{a} \rho+\left(a^{2}-b^{2}\right) r \Sigma_{b} \sin ^{2} \theta}{\Sigma_{a}^{2} \Sigma_{b}^{2}-r^{2} \rho^{2}} .
\end{aligned}
$$

This polynomial is the square of a polynomial of degree 2. Therefore, the MP metric has Petrov type $\underline{22}$.

\section{Conclusions and topics for further research}

In this article, we showed that the five-dimensional MP metric has the same Petrov type as its static limit, namely Petrov type $\underline{22}$. Some open problems are the following.

- Recently, Emparan and Reall found a black rotating ring [3], see ref. [4] for easier coordinates. It would be nice to know its Petrov type.

- In four dimensions, adding electric charge to the rotating star does not change its Petrov type; the Kerr-Newmann metric has Petrov type $D$. In five dimensions, the story is more complicated. The metric of an electrically charged rotating black hole is only known in five dimensions when there is a specific Chern-Simons term in the action. The particular form of this Chern-Simons term is dictated by supersymmetry. The charged

rotating black hole in this theory is described by the BMPV metric [5], which was found 
by using duality. It would be good to calculate its Petrov type. The charged rotating black hole is not known when this Chern-Simons term has an arbitrary (or even zero) coefficient. It remains to be seen if it can be found within the class of algebraically special metrics.

\section{Acknowledgments}

This work has been supported in part by the NSF grant PHY-0098527.

\section{References}

[1] R. C. Myers and M. J. Perry, "Black Holes In Higher Dimensional Space-Times," Annals Phys. 172, 304 (1986).

[2] P. J. De Smet, "Black holes on cylinders are not algebraically special," Class. Quant. Grav. 19, 4877 (2002) [arXiv:hep-th/0206106].

[3] R. Emparan and H. S. Reall, "A rotating black ring in five dimensions," Phys. Rev. Lett. 88, 101101 (2002) [arXiv:hep-th/0110260].

[4] K. Hong and E. Teo, "A new form of the C-metric," Class. Quant. Grav. 20 (2003) 3269 [arXiv:gr-qc/0305089].

[5] J. C. Breckenridge, R. C. Myers, A. W. Peet and C. Vafa, "D-branes and spinning black holes," Phys. Lett. B 391 (1997) 93 [arXiv:hep-th/9602065]. 